\title{
Impacto de la lectura repetida interactiva en las habilidades verbales de preescolares de contextos vulnerables
}

\author{
Pelusa Orellana García \\ orcid.org/oooo-0003-3851-8780 \\ Universidad de los Andes, Santiago, \\ Chile \\ porellan@uandes.cl
}

\author{
María Francisca Valenzuela \\ orcid.org/o000-0002-7019-9110 \\ Universidad de los Andes, Santiago, \\ Chile \\ mvalenzuela@uandes.cl
}

\section{Kattia Muñoz \\ orcid.org/00oo-0o02-2760-7043 \\ Universidad de los Andes, Santiago, \\ Chile \\ kmunoz@uandes.cl}

\section{Resumen}

Este estudio describe la implementación de la lectura repetida interactiva, como actividad para incrementar el vocabulario y la conciencia semántica de preescolares chilenos de contextos vulnerables. Estudios en este país muestran que son escasas las instancias en que se promueve el vocabulario en sala de clases preescolares. En esta investigación participaron 47 estudiantes de prekinder divididos en grupos experimental y control. Maestras y asistentes del grupo experimental recibieron una capacitación en el uso de la lectura repetida interactiva, que incluyó definiciones conceptuales, demostraciones, acompañamiento, libros para la lectura y material de actividades. El grupo de control recibió la intervención, una vez finalizado el estudio. Se examinaron los resultados de los alumnos mediante análisis descriptivos y de varianza, con mejoras significativas en vocabulario receptivo, pero no en habilidades metasemánticas, como el uso de analogías, identificación de textos sin sentido y semejanzas y diferencias a favor del grupo experimental. El estudio también mostró que la combinación en conjunto de estrategias didácticas orales e icónicas favorece la adquisición de estas mejoras.

\section{Palabras clave (fuente: tesauro de la UNESCO)}

Alfabetización; educación de la primera infancia; comprensión; lectura; lenguaje; lenguaje hablado; vocabulario.

Recepción: 25/02/2018 | Enviado a pares: 11/08/2018 | Aceptación por pares: 10/11/2018 | Aprobación: 11/11/2018 


\title{
Impact of Repeated Interactive Read-aloud on the Speaking Skills of Preschool Students in Vulnerable Contexts
}

\begin{abstract}
This study describes the implementation of repeated interactive read-aloud, as an activity to increase the vocabulary and semantic awareness of Chilean preschool students in vulnerable contexts. This research involved 47 prekindergarten students, divided into the experimental and control groups. Teachers and assistants of the experimental group received training in the use of repeated interactive read-aloud, which included conceptual definitions, demonstrations, assistance, reading books and activity materials; the control group received only the reading books. The results of students were compared by means of descriptive and variance analyses. Significant improvements in receptive vocabulary were observed in the experimental group, but not in meta-semantic skills, such as the use of analogies, identification of meaningless texts, and similarities and differences. The study concluded that the whole combination of speaking and iconic teaching strategies benefits the acquisition of receptive vocabulary.
\end{abstract}

Keywords (source: UNESCO Thesaurus)

Literacy; early childhood education; comprehension; reading; language; spoken language; vocabularies. 


\section{Impacto da leitura repetida interativa nas habilidades verbais de estudantes pré-escolares em contextos vulneráveis}

\section{Resumo}

Este estudo descreve a implementação da leitura repetida interativa como atividade para incrementar o vocabulário e a consciência semântica de estudantes pré-escolares chilenos em contextos vulneráveis. Nesta pesquisa participaram 47 estudantes da pré-escola, divididos nos grupos experimental e controle. Professoras e assistentes do grupo experimental receberam uma capacitação no uso da leitura repetida interativa, que incluiu definições conceituais, demonstrações, acompanhamento, livros para a leitura e material de atividades; o grupo de controle recebeu apenas os livros de leitura. Os resultados dos alunos foram comparados mediante análises descritivas e de variância. Foram observadas melhoras significativas no vocabulário receptivo a favor do grupo experimental; mas não em habilidades metasemânticas, como o uso de analogias, identificação de textos sem sentido e semelhanças e diferenças. $O$ estudo concluiu que a combinação de estratégias didáticas orais e icônicas favorece a aquisição do vocabulário receptivo.

\section{Palavras-chave (fonte: tesauro da UNESCO)}

Alfabetização; educação infantil; compreensão; leitura; linguagem; linguagem falada; vocabulário. 
Numerosos estudios han documentado la importancia e impacto del desarrollo de lenguaje en los primeros años de vida (Pianta, 2012; Lawrence y Snow, 2011; Connor y Craig, 2006), y la lectura de cuentos ha sido identificada como un excelente escenario para favorecerlo, pues permite trabajar de manera más profunda el vocabulario y la comprensión en un contexto lingüístico específico y auténtico a la vez (Wasik y Bond, 2001; Justice y Kaderavek, 2004). Por otra parte, la lectura en voz alta es una actividad ampliamente utilizada en la educación inicial en muchos países (Neslihan y Simsek, 2014), pero la evidencia sugiere que el solo hecho de leer cuentos a los niños no es suficiente para favorecer el desarrollo del vocabulario y la comprensión oral. La forma en que se planifican las actividades y se leen los cuentos afecta dichos procesos (McGee y Schickedanz, 2007; Strasser y Lissi, 2009). La lectura en voz alta puede promover el lenguaje a través de la interacción lingüística que se genere con la lectura (Hoffman, 2011), si es que se utiliza de manera sistemática y si las actividades que se ofrecen antes, durante y después de la lectura son cuidadosamente planificadas (Otto, 2008).

Las experiencias en alfabetización en la primera infancia buscan compensar las falencias en la exposición y/o la falta de experiencias que los niños provenientes de sectores socioeconómicos desaventajados traen a la escuela y que pueden tener efectos negativos para el aprendizaje de la lectura y escritura al ingreso a la educación formal (Hart y Risley, 1995; Neuman y Celano, 2001; Fitzgerald, Spiegel y Cunningham, 1991). La exposición a distintos tipos de texto y la conversación que de ello surge son insumos para interacciones que tengan al lenguaje como eje central (OECD, 2013). En realidad, parte de las brechas en el desempeño pueden atribuirse al escaso desarrollo de habilidades de lenguaje y de alfabetización (Neuman, 2003; Burns, Griffin y Snow, 1999). Esto es particularmente importante en países en desarrollo, en los cuales los factores asociados a la educación parental, el tipo y la calidad de las interac- ciones verbales padre-hijo y la lectura compartida de cuentos determinan el repertorio lexical, sintáctico y pragmático de los niños (Lennox, 2013; Treviño, 2013).

La lectura de cuentos en primera infancia tiene considerables beneficios para el desarrollo cognitivo y lingüístico. En el caso de la lectura que los padres realizan con sus hijos, estudios muestran que esta clase de experiencias puede explicar hasta el 8\% de la varianza en incremento verbal, alfabetización emergente y habilidades lectoras de los niños (Bus, van Ijzendoorn y Pelligrini, 1995). Estas instancias son también propicias para incorporar aspectos importantes de la enseñanza de la lectura (Allor y McCathren, 2003; Justice, Kaderavek, Fan, Sofka y Hunt, 2009; Wiseman, 2011; Santoro, Chard, Howard y Baker, 2008), como son la promoción del uso de habilidades del pensamiento en niveles cognitivos más desafiantes (Justice et al., 2009), el fomentar la argumentación a través de la discusión de cuentos y la valoración de la lectura en general (Cunningham y Stanovich, 1997; Baker, Scher y Mackler, 1997).

\section{Características y efectividad de una lectura repetida interactiva}

En la lectura repetida interactiva un adulto lee a un grupo de niños un mismo cuento tres veces en una semana, pero de maneras distintas (Smolkin y Donovan, 2001; Bortnem, 2008), respecto del cual se discute usando preguntas abiertas y focalizando la atención en palabras de vocabulario que aparecen en el cuento. La primera lectura es más pausada y la conversación se centra en analizar los personajes del cuento. El profesor aprovecha para explicar en forma sencilla y amigable (Beck y McKeown, 2007) el significado de tres o cuatro palabras nuevas, esenciales para comprender el texto. Se utiliza la discusión dialógica para conversar acerca de los distintos componentes del cuento, apoyando a los niños en la elaboración de sus propias ideas, la expresión de las mismas y la capacidad de escucharse unos a otros (Lennox, 2013). La segunda lectura generalmente se realiza poniendo mayor énfasis en desarrollar 
un pensamiento más complejo y un conocimiento literario más sofisticado (Copenhaver-Johnson, Bowman y Rietschlin, 2009). Finalmente, la tercera lectura del cuento apunta a que los alumnos sean capaces de elaborar discursos argumentativos fundamentando sus opiniones en el texto e incorporando las nuevas palabras en sus expresiones. Las preguntas que el profesor formula en esta tercera lectura corresponden a niveles taxonómicos superiores, como es el pensamiento inferencial, la síntesis o la evaluación.

A través de la lectura repetida interactiva se pueden adquirir muchas habilidades del lenguaje y la lectura, entre ellas, la comprensión oral (Dickinson y Smith, 1994), el conocimiento de vocabulario (Kucan, 2012), la formulación de preguntas más elaboradas, la capacidad de predecir y relacionar conocimientos previos. En el caso de la lectura repetida interactiva, estas instancias son usadas sistemáticamente durante la semana, y los alumnos leen y discuten el mismo texto dos o tres veces durante el mismo período, lo que ayuda a consolidar las habilidades en el contexto de una lectura que se hace más familiar a los alumnos y que, por tanto, favorece su adquisición.

Asimismo, se han documentado los beneficios de la lectura repetida interactiva para promover el desarrollo del lenguaje, el pensamiento y la alfabetización (Dickinson, 2001; Hargrave y Sénéchal, 2000; Paris y Paris, 2003). Precisamente el hecho de que esta lectura requiera de una participación mucho más activa por parte de los alumnos hace de ella una instancia propicia para que se generen conversaciones más extendidas en el tiempo. Como afirma Dickinson (2001), la lectura en voz alta es más efectiva cuando se involucra activamente a los niños, formulando preguntas y haciendo predicciones, más que escuchando pasivamente. Esta estrategia requiere que el profesor modele pensamiento de alta calidad, conversaciones analíticas y la comprensión de nuevo vocabulario (McGee y Schickedanz, 2007).
Por otra parte, Beck, McKeown y Kucan (2001) plantean la importancia de centrar la atención en algunas palabras de vocabulario escogidas previamente, a partir de definiciones simples que puedan ser comprendidas fácilmente por los niños. Dado que la lectura se repite en tres instancias, las palabras nuevas son utilizadas en múltiples ocasiones, lo que ayuda a que estas se incorporen al vocabulario activo de los estudiantes. Por ejemplo, después de que se explica el significado de las nuevas palabras, los alumnos aprenden a utilizarlas en otros contextos, más allá del texto leído. Las autoras también siguieren formular preguntas abiertas que amplíen el espectro de posibles respuestas, para así favorecer la construcción de un diálogo sostenido en el tiempo.

Un aspecto importante de la lectura repetida interactiva es la selección de libros. El docente debe dedicar un tiempo considerable a escoger literatura de calidad, a partir de una amplia gama de géneros, temas y niveles de complejidad textual, para asegurar que se genere una conversación rica en ideas y lenguaje. El uso de textos informativos, por ejemplo, presenta a los alumnos un texto mucho más elaborado que puede favorecer el desarrollo de un diálogo más sofisticado (Anderson et al., 2012). Según Lennox (2013), la selección del texto adecuado contribuye al éxito de la lectura repetida interactiva y el tipo de libro que se escoge determina la calidad y el tipo de conversación que se genera. De la misma manera, la planificación de cada una de las instancias de lectura repetida interactiva debe realizarse en forma meticulosa, considerando el objetivo, el momento y lugar en el que se llevará a cabo, los conocimientos previos de los estudiantes y los aspectos formales del cuento que pudieran gatillar niveles más profundos de conversación, utilizando, por ejemplo, las ilustraciones, el nombre del autor o la temática del cuento para incitar a los alumnos a conversar sobre el mismo (Otto, 2008). Durante la lectura del cuento en voz alta, el profesor debe preocuparse de su lenguaje no verbal y de cómo este promueve la comprensión del texto: tono de voz, postura, pausas, 
elaboración de ideas, tiempo para promover el diálogo entre los estudiantes, etc.

La lectura repetida interactiva tiene un formato relativamente estructurado. Como se mencionó anteriormente, un mismo texto se lee tres veces durante una semana y a través de esas lecturas se trabaja el vocabulario y la comprensión de manera más específica. El profesor debe establecer un andamiaje para que los niños comprendan mejor y ha de modelar el uso de inferencias, explicaciones y nuevas palabras o la formulación de predicciones (Robbins y Ehri, 1994; Morrow y Smith, 1990; Duke y Kays, 1998; Hargrave y Sénéchal, 2000). Mediante la interacción repetida con un texto, los alumnos internalizan estas habilidades y luego las proyectan hacia otras instancias de comprensión (Barrentine, 1996). Al discutir el uso de las nuevas palabras, ellos son capaces de incorporarlas a su repertorio de manera más efectiva (Blewitt, Rump, Shealy y Cook, 2002; Wasik y Bond, 2001; Beck, McKeown y Kucan, 2002).

La incorporación de la lectura repetida interactiva como actividad de alfabetización en la educación temprana ha mostrado ganancias importantes en diversos aspectos de su desarrollo, aunque más específicamente en lo que respecta al vocabulario (Hargrave y Sénéchal, 2000) y la comprensión (Wiseman, 2011; Van den Broek, 2001). También se han observado incrementos en áreas tales como el interés de los alumnos por los libros (Bortnem, 2008), el concepto de lo impreso (Sulzby y Teale, 1991), la capacidad de comprender lo que se escucha y la calidad de las interacciones dialógicas (Reutzel, 2001; Dickinson y Smith, 1994), y las habilidades de decodificación (Feitelson, Kita y Gloldstein, 1986). En Chile existen estudios que han demostrado la alta correlación entre las interacciones verbales y el desarrollo posterior de la lectura, pero muy pocos se han centrado en investigar el rol de la lectura y su relación con aspectos tempranos del lenguaje y la alfabetización (Eyzaguirre y Fontaine, 2008; Larraín, Strasser y Lissi, 2012; Valenzuela, 2005). En ellos se muestra que en las aulas preescolares casi no existe el diálogo exploratorio, hay escaso tiempo para que los alumnos expresen sus ideas y las interacciones verbales son siempre iniciadas y controladas por el docente. En cuanto a las preguntas que se formulan, estas son por lo general de tipo literal, y no se observa evidencia de intencionalidad en la enseñanza explícita de vocabulario (Eyzaguirre y Fontaine, 2008; Valenzuela, 2005; Strasser, Lissi y Silva 2009).

En los países en vías de desarrollo la enseñanza explícita y sistemática de la alfabetización muchas veces no está en el corazón de las experiencias preescolares. De hecho, sabemos poco acerca del impacto que tiene la lectura de cuentos en el desarrollo del lenguaje infantil (Strasser y Lissi, 2009; Rolla, Arias y Villers, 2005). En Chile, por ejemplo, las investigaciones muestran que se dedica muy poco tiempo a la lectura de cuentos y a la enseñanza del lenguaje (Eyzaguirre y Fontaine, 2008). El nivel de desarrollo del lenguaje infantil se considera inferior a lo que, desde un punto de vista evolutivo, correspondería a dicho grupo etario (Villalón, Suzuki, Herrera y Mathiesen, 2002), y el nivel de desempeño muestra una casi nula variación como consecuencia de asistir o no al jardín infantil. Resultados similares se han encontrado en estudios de tipo longitudinal (e.g., Mineduc, 1998), indicando que no hay crecimiento en las habilidades cognitivas o de alfabetización al finalizar el primer grado. Larraín, Strasser y Lissi (2012) analizaron el impacto de la lectura compartida de cuentos en el aprendizaje del vocabulario a partir de dos estudios realizados con preescolares en contextos de riesgo social. En un estudio compararon la enseñanza explícita de palabras nuevas a través de la lectura compartida con y sin definiciones explícitas de las palabras nuevas. Descubrieron que, pese a que la definición elaborada de las nuevas palabras tenía un impacto positivo en el aprendizaje de vocabulario, la brecha entre quienes tienen mayor y menor repertorio léxico se mantiene. También observaron que el uso de definiciones más sencillas de las palabras nuevas parece ser más efectivo para su adquisición. 
La asistencia a la educación preescolar ha tenido algunos impactos positivos en términos cognitivos para los niños chilenos de hogares muy desaventajados (e.g., Bedregal, 2006; Urzúa y Veramendi, 2011; Noboa y Urzúa, 2012). Bedregal encontró mejoras significativas en el lenguaje oral de los niños y una mejor adaptabilidad al entorno escolar para los niños de hogares en riesgo social que asistían a la educación preescolar de manera más sistemática. Sin embargo, la mayoría de los estudios longitudinales solo ha examinado efectos de la educación preescolar en el corto plazo y en zonas urbanas específicas. Pocos estudios se han centrado en analizar el impacto de intervenciones, especialmente en el desarrollo del lenguaje y la alfabetización.

La calidad de la enseñanza a nivel preescolar también muestra hallazgos interesantes en relación con el grado en el que el lenguaje y la alfabetización se incorporan de manera formal y explícita en la enseñanza y el aprendizaje. En Chile, por ejemplo, las educadoras de párvulos son reacias a enseñar lenguaje oral, comprensión o vocabulario de manera intencionada. Pese a que el marco curricular chileno entrega bastante libertad a los docentes para estructurar, secuenciar y elegir los contenidos, habilidades y actividades que desarrollan (Peralta, 2011; Yoshikawa et al., 2015), se observa también un fuerte temor a que el centrarse en habilidades y enseñar de manera explícita sea considerado como una escolarización excesiva que no es el objetivo de la educación inicial. Por lo mismo, los docentes muchas veces no planifican su enseñanza teniendo en mente objetivos de lenguaje y alfabetización, y las actividades de lectura de cuentos son percibidas como momentos de recreación, más que como vehículos explícitos para promover el pensamiento dialógico o el desarrollo lingüístico de los alumnos.

Por otra parte, la calidad del entorno letrado de las salas de educación preescolar en Chile es baja y se constata que existen diferencias significativas, si se analiza por nivel socioeconómico de los estudiantes que asisten a los distintos centros. En términos de actividades de lectura, en un $40 \%$ de las 147 salas evaluadas, las educadoras dedicaban 5 minutos o menos a la lectura grupal de cuentos (Orellana y Melo, 2014).

Las preguntas de investigación que se busca responder en el presente estudio son las siguientes: 1) ¿puede una intervención de lectura repetida interactiva de seis semanas mejorar significativamente las habilidades verbales de los preescolares, especialmente en vocabulario? y 2) ¿cuáles habilidades verbales denotan mayores ganancias?

\section{Métodos}

Diseño. El presente estudio es de carácter cuasiexperimental y compara el desempeño de estudiantes preescolares de un grupo experimental en una serie de habilidades verbales respecto de sus pares en un grupo de control. La intervención a la que estuvo sujeto el grupo experimental consistió en el uso por parte de las docentes de la lectura repetida interactiva durante seis semanas. Para ello se diseñó un modelo de intervención que incluyó la capacitación a las educadoras del grupo experimental, el modelamiento de las estrategias, la entrega y descripción del material para realizar la lectura repetida interactiva durante las sesiones de intervención, y el acompañamiento y retroalimentación durante el proceso.

Participantes. Un total de 47 alumnos (edad promedio 46,3 meses) y 4 educadoras de párvulos pertenecientes a dos jardines infantiles de escasos recursos en una zona semirrural de la Región Metropolitana de Santiago participaron en este estudio. Ambos establecimientos están ubicados en una misma comuna, a pocos metros de distancia el uno del otro. La edad promedio de los alumnos en el grupo experimental era de 47 meses, mientras que la edad promedio de los alumnos del grupo de control era de 45 meses. Las pruebas no paramétricas de muestras independientes de Mann-Whitney $U$ revelaron que la distribución de los puntajes en los grupos era normal para todas las variables, salvo el vocabulario al inicio de la intervención. 
El jardín infantil que se seleccionó como grupo experimental es un establecimiento particular subvencionado, y como tal recibe una porción de financiamiento estatal, pero es administrado por una fundación. El jardín correspondiente al grupo de control es administrado por la municipalidad y es enteramente financiado con fondos estatales. Ambos jardines infantiles atienden a los niños en forma gratuita. En términos sociodemográficos, ambos establecimientos reciben a niños en situación de riesgo social. Las educadoras de ambos establecimientos tienen un perfil similar en términos de estudios superiores y años de experiencia. Todas tenían estudios universitarios de pregrado en el área de educación preescolar, y dos pertenecientes al grupo experimental se encontraban estudiando un magister a distancia.

Para todos los participantes se obtuvo consentimiento informado previo al inicio del estudio. Al finalizar la investigación, y considerando las implicancias éticas del diseño, las educadoras del grupo experimental capacitaron a sus pares del grupo de control en el uso de la lectura repetida interactiva, a fin de que esos grupos pudieran conocerla y utilizarla también.

Datos. El estudio incluyó la recolección de datos numéricos, para los cuales se tomaron pruebas pre y posintervención a todos los alumnos. Se utilizaron los siguientes instrumentos de evaluación:1) Prueba para Habilidades Metalingüísticas del tipo Semántico (PHMS) 4 a 6 años, desarrollada por Renz, Valenzuela y Yakuba (2014), la cual incluye la evaluación de habilidades semánticas en dos modalidades: oral (en que el niño contesta preguntas relacionadas con categorización de palabras, adivinanzas, completación de analogías, reconocimiento de absurdos y semejanzas y diferencias) e icónico (en que se debe marcar la respuesta correcta en tareas de categorización, adivinanzas y analogías); y 2) el TEVI-R (Test de Vocabulario en Imágenes, de Echeverría, Herrera y Segure, 2002), el cual ha sido elaborado para eva- luar el vocabulario receptivo en la población escolar entre 2 años y medio y 17 años de edad. Este instrumento consiste en un set de 116 láminas, cada una con 4 ilustraciones que representan sustantivos, verbos y adjetivos, y una hoja de protocolo. El alumno debe indicar el dibujo correspondiente al estímulo que es dado por el evaluador. La prueba TEVI fue adaptada para ser aplicada en formato digital por Orellana, Melo y Fitzgerald (2014). En este formato, los niños escuchan el estímulo ("muéstrame casa") y marcan con su dedo en la pantalla de una Tablet el dibujo correspondiente a la palabra escuchada. La validez y confiabilidad de este formato de aplicación ha sido establecida en estudios previos (e.g. Orellana, Melo y Fitzgerald, 2014).

LA INTERVENCIÓN. Las educadoras del grupo experimental recibieron una capacitación sobre cómo implementar la lectura repetida interactiva en el aula. La capacitación incluyó la definición de la actividad, sus características, etapas, ventajas, así como también un modelo de planificación para que las educadoras siguieran durante la lectura de cuentos. La capacitación se estructuró en cuatro sesiones con actividades asociadas para tres días a la semana cada una, con base en un cuento distinto cada semana. Se explicaron las siguientes tres etapas por semana para implementar la estrategia: 1) conocimiento del cuento: su vocabulario con definición amigable, preguntas literales y adivinanzas; 2) comprensión a través de preguntas: preguntas inferenciales y categorización de palabras; y 3) reconstrucción de la historia guiada: recuento secuencial con uso de palabras aprendidas e inferencias. Cada sesión fue elaborada de manera detallada por expertos en lenguaje y los documentos de las mismas fueron entregados a cada educadora participante de la intervención de manera que pudiesen internalizar el trabajo que se realizaría y así facilitar su implementación.

Durante el período de intervención las educadoras aplicaron la estrategia 3 veces por semana durante 6 semanas, correspondiendo un cuento di- 
ferente para cada semana, y actividades diferentes para cada una de ellas. Al hacerlo las educadoras filmaron al menos 1 lectura por cada 2 semanas. Dichas filmaciones eran enviadas al grupo investigador, para poder dar retroalimentación. Las investigadoras visitaron el jardín infantil 3 veces durante el período de intervención, con el fin de conversar sobre el desarrollo de la actividad, retroalimentar y asegurar la correcta implementación de la estrategia. En la séptima semana se realizó la toma de evaluaciones posintervención en ambos jardines infantiles, las cuales fueron posteriormente procesadas para obtener estadísticas descriptivas (Tabla 1). Un equipo de evaluadoras debidamente entrenado se encargó de aplicar las pruebas en ambos períodos.

AnálISIS. Para determinar el impacto de la intervención antes descrita se utilizaron métodos estadísticos descriptivos y análisis de varianza. Se utilizó el software SPSS en su versión 23 para el análisis de los datos. En el presente estudio solo se da cuenta de los resultados obtenidos por los alumnos evaluados y no del análisis de las filmaciones de las propias educadoras en su implementación de la estrategia. El análisis de las filmaciones se utilizó para asegurar la fidelización de la implementación de la misma.

\section{Resultados}

La Tabla 1 muestra las estadísticas descriptivas para ambos grupos, antes y después de la intervención. En general, los alumnos mejoraron su desempeño en la prueba PHMS, pasando de 30,4 a 32,6 puntos después de 6 semanas. El máximo puntaje en la prueba PHMS es de 80 puntos, de los cuales 50 son asignados a la parte oral y 30 a la icónica. El puntaje promedio obtenido los ubica aún en el tercio inferior en cuanto a habilidades metalingüísticas de tipo semántico. En cuanto a vocabulario receptivo, este se incrementó de 24,5 (50 puntaje T) a 33,2 (66 puntaje T). De acuerdo con las normas de la prueba TEVI, y considerando la edad promedio del grupo evaluado, se pasó de la categoría "normal" (45 a 54 puntos) a la categoría "sobresaliente" (sobre 65 puntos).

Como es posible observar en la Tabla 1, los alumnos del grupo experimental, en promedio, obtienen un resultado superior al del grupo de control en ambas evaluaciones. En el caso de la prueba de vocabulario receptivo, los alumnos en el grupo de control obtienen un resultado casi 6 puntos superior al grupo experimental. Es importante recordar que el grupo experimental es 2 meses mayor que el grupo de control y que, tal como se observaba en las pruebas no paramétricas de Mann-Whitney $U$, la distribución de los puntajes en vocabulario al inicio del estudio difiere significativamente entre los grupos. Pese a ello, los resultados en vocabulario receptivo posintervención mostraron que no había diferencias significativas en la distribución de los puntajes dentro de ambos grupos, pero la diferencia entre los resultados del grupo experimental y el de control en la prueba de vocabulario sí fue significativa: $F(1,46)=5,882 p<0,05$. Para la prueba PHMS, la diferencia entre los grupos es menor de 1 punto en el resultado promedio, y no se observaron diferencias estadísticamente significativas en la distribución de puntajes pre y posintervención.

Tabla 1. Estadisticas descriptivas por grupo

\begin{tabular}{|c|c|c|c|c|c|}
\hline Grupo & $\begin{array}{c}\text { Edad } \\
\text { (meses) }\end{array}$ & $\begin{array}{c}\text { Vocab } \\
\text { (pre) } \\
\text { M(SD) }\end{array}$ & $\begin{array}{l}\text { Vocab } \\
\text { (pos) } \\
\text { M(SD) }\end{array}$ & $\begin{array}{l}\text { PHMS } \\
\text { (pre) } \\
\text { M(SD) }\end{array}$ & $\begin{array}{c}\text { PHMS } \\
\text { (pos) } \\
\text { M(SD) }\end{array}$ \\
\hline 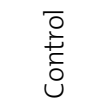 & 45 & $\begin{array}{c}21,6 \\
(10,4)\end{array}$ & $\begin{array}{l}28,7 \\
(9,7)\end{array}$ & $\begin{array}{c}31 \\
(6,06)\end{array}$ & $\begin{array}{l}32,3 \\
(7,03)\end{array}$ \\
\hline 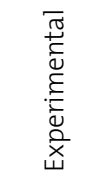 & 47 & $\begin{array}{l}30,9 \\
(12,2)\end{array}$ & $\begin{array}{l}35,9 \\
(12,1)\end{array}$ & $\begin{array}{c}30,07 \\
(5,7)\end{array}$ & $\begin{array}{l}32.7 \\
(4,7)\end{array}$ \\
\hline
\end{tabular}

Fuente: elaboración propia. 
ISSN 0123-1294 | e-ISSN 2027-5358 | Educ.Educ. Vol. 21. No. 3 | Septiembre-diciembre de 2018 | pp. 409-432.

Universidad de La Sabana | Facultad de Educación

Dado que la prueba PHMS evalúa distintas habilidades de tipo metalingüístico, interesaba analizar el desempeño de los estudiantes en cada una de las subpruebas. La Tabla 2 desglosa estos resultados, además, según el tipo de estímulo que se le entregó al niño (verbal o icónico).

Tabla 2. Estadisticas descriptivas para prueba PHMS (pre y pos)

\begin{tabular}{|c|c|c|c|c|}
\hline Grupo experimental & Mínimo & Máximo & $M$ & SD \\
\hline TOTAL PHMS (pre) & 15 & 37 & 29,5 & 5,9 \\
\hline TOTAL PHMS (pos) & 16 & 54 & 32,9 & 5,8 \\
\hline Verbal Categorías (pre) & $\mathrm{O}$ & 9 & 4,1 & 1,8 \\
\hline Verbal Categorías (pos) & O & 10 & 4,7 & 1,6 \\
\hline Verbal Analogías (pre) & 0 & 7 & 4,1 & 1,7 \\
\hline Verbal Analogías (pos) & 1 & 9 & 4,3 & 1,5 \\
\hline Verbal Absurdo (pre) & 1 & 9 & 5,2 & 1,6 \\
\hline Verbal Absurdo (pos) & 2 & 8 & 5,3 & 1,2 \\
\hline Verbal Adivinanzas (pre) & 2 & 5 & 4,0 & 1,3 \\
\hline Verbal Adivinanzas (pos) & 2 & 6 & 4,5 & 1,0 \\
\hline Icónico Categorías (pre) & $\mathrm{O}$ & 8 & 3,9 & 1,9 \\
\hline Icónico Categorías (pos) & o & 10 & 4,7 & 1,6 \\
\hline Icónico Analogías (pre) & o & 8 & 3,8 & 2,0 \\
\hline Icónico Analogías (pos) & $\mathrm{O}$ & 8 & 4,4 & 1,8 \\
\hline Icónico Adivinanzas (pre) & o & 7 & 4,1 & 1,7 \\
\hline Icónico Adivinanzas (pos) & 0 & 8 & 4,6 & 1,4 \\
\hline Grupo de control & Mínimo & Máximo & $M$ & SD \\
\hline TOTAL PHMS (pre) & 16 & 35 & 29,93 & 5,6 \\
\hline TOTAL PHMS (pos) & 16 & 37 & 32,77 & 4,5 \\
\hline Verbal Categorías (pre) & 1 & 9 & 4,29 & 2,48 \\
\hline Verbal Categorías (pos) & 2 & 7 & 4,74 & 1,26 \\
\hline Verbal Analogías (pre) & o & 6 & 4,23 & 1,54 \\
\hline Verbal Analogías (pos) & 1 & 6 & 4,16 & 1,53 \\
\hline Verbal Absurdo (pre) & 1 & 9 & 5,29 & 1,65 \\
\hline Verbal Absurdo (pos) & 3 & 8 & 5,32 & 1,04 \\
\hline Verbal Adivinanzas (pre) & 2 & 5 & 4,13 & 1,33 \\
\hline Verbal Adivinanzas (pos) & 2 & 6 & 4,48 & 1,05 \\
\hline Icónico Categorías (pre) & o & 8 & 3,94 & 1,98 \\
\hline Icónico Categorías (pos) & o & 8 & 4,81 & 1,30 \\
\hline Icónico Analogías (pre) & o & 8 & 4,16 & 1,95 \\
\hline Icónico Analogías (pos) & o & 8 & 4,35 & 1,85 \\
\hline Icónico Adivinanzas (pre) & o & 7 & 4,26 & 1,67 \\
\hline Icónico Adivinanzas (pos) & o & 8 & 4,56 & 1,85 \\
\hline
\end{tabular}

Fuente: elaboración propia. 
Como se ve en la Tabla 2, el mayor incremento de puntaje posterior a la intervención se observa para las categorías, tanto en lo icónico como en lo verbal, y tanto para el grupo experimental como para el de control. En ambos grupos la magnitud del incremento es mayor en las categorías icónicas (0,8 y o,9 respectivamente) que en las verbales ( 0,6 y 0,5 respectivamente). En segundo lugar, se observa un incremento importante en las analogías y adivinanzas en formato icónico del grupo experimental, que se incrementan en o,6 y 0,5 puntos respectivamente. En el grupo control, estas habilidades aumentan, pero en una proporción menor (o,2 y o,3). En el caso de las analogías, absurdo y adivinanzas en formato verbal, los niños obtienen un mejor desempeño en adivinanzas. Los niños del grupo experimental mejoran en las tres áreas, mientras que en el grupo de control el puntaje promedio disminuye en el área de analogías y aumenta en absurdo y adivinanzas, aunque en menor proporción que en el grupo experimental. Los análisis de varianza, sin embargo, muestran que no existen diferencias significativas en el desempeño de los grupos para ninguna de las habilidades evaluadas. Esto se explica por la magnitud del incremento, que es relativamente pequeña en ambos grupos.

\section{Discusión}

En el presente estudio se intentó determinar si la implementación de la lectura repetida interactiva como estrategia de lectura de cuentos podría impactar positivamente el desarrollo del vocabulario y las habilidades metalingüísticas de tipo semántico en niños preescolares provenientes de contextos de pobreza. Respecto de la primera pregunta de investigación, los resultados indican que solo el incremento en el vocabulario receptivo de los alumnos evidenció diferencias estadísticamente significativas entre los grupos. Si bien se observan mejoras en habilidades verbales de tipo semántico, las ganancias observadas corresponden al vocabulario receptivo y no al productivo, al menos en las habilidades metasemánticas que evalúa la PHMS.
Estos resultados están en línea con estudios como el de Kindle (2009), en el que se entrega evidencia de que la lectura repetida interactiva es una herramienta eficaz para enseñar palabras de vocabulario a niños pequeños. Similar resultado obtuvo Leung (2008), quien además incluyó actividades kinestésicas y el uso de la expresión verbal como una estrategia para que los alumnos explicaran el significado de las palabras nuevas. Cabe recordar que las palabras elegidas para ser enseñadas por las educadoras corresponden a lo que Beck et al. (2002) denominan nivel 2 y que corresponde a aquellos conceptos que los alumnos pueden conocer y comprender vagamente o no conocer del todo, pero que son fundamentales para comprender un texto. Pearson, Hiebert y Kamil las definen como "parte del discurso académico sofisticado" (2007, p. 291). Al igual que Leung, las educadoras del presente estudio enseñaron a los alumnos una serie de técnicas para demostrar dominio del significado de una palabra. Por ejemplo, usaron material concreto para explicitar el significado, contextualizaron las definiciones, dieron ejemplos y no ejemplos de cada una de las palabras, pidieron a los niños usar las palabras en frases y adivinanzas, extendiendo el uso de las palabras más allá del contexto del cuento leído. Al respecto, los resultados del presente estudio muestran que los alumnos obtuvieron mejores resultados en los ítems icónicos de la prueba PHMS que en los verbales. Ello refleja que, así como la enseñanza del vocabulario resulta más efectiva cuando se realiza integrando distintos "medios" (Shatz y Baldwin, 1986; Beck et al., 2002), la evaluación del vocabulario usando el medio icónico puede favorecer la consolidación de la adquisición del significado de las palabras aprendidas (Beck y McKeown, 2007). El apoyo visual ha demostrado ser una herramienta eficaz, especialmente en niños pequeños cuyo repertorio léxico está aún en desarrollo o que han tenido poca exposición a lenguaje oral más sofisticado.

Por otra parte, llama la atención la diferencia observada en el logro alcanzado en la Prueba TEVI 
versus los resultados obtenidos por los alumnos en la PHMS. Al respecto, es importante recordar que la primera evalúa vocabulario receptivo, es decir, permite saber si el evaluado conoce el significado de una palabra, aunque no sea capaz de utilizarla en una oración u otro contexto. Corresponde, por tanto, a una especie de lexicón mental o grupo de conceptos acerca de los cuales el hablante tiene una idea vaga de su significado, la cual es activada mediante estímulos visuales. El vocabulario activo o productivo, en cambio, es aquel grupo de palabras cuyo significado el hablante conoce y puede utilizar. En la prueba PHMS se pide a los alumnos realizar una serie de tareas (categorizar, completar adivinanzas y analogías, identificar absurdos y establecer semejanzas y diferencias) que requieren de un conocimiento del significado mucho más profundo y aplicado, pues el alumno debe saber usar la palabra en una frase que sea verosímil, compararla con otros términos o establecer relaciones, como es el caso de la analogía. Por lo anterior, es posible que la diferencia en el puntaje obtenido en ambas pruebas difiera de manera importante, aun cuando ambas evalúan el mismo constructo de conocimiento semántico.

Para responder a la segunda pregunta de investigación, se analizaron con mayor detenimiento los resultados de la prueba PHMS, pues ella evalúa distintos aspectos metasemánticos del lenguaje con mayor profundidad. Además de las mejoras en vocabulario receptivo, interesaba determinar en qué grado la lectura repetida interactiva promovía el desarrollo de habilidades expresivas del lenguaje, vinculadas a la adquisición e incorporación de nuevas palabras en el léxico propio. Si bien los resultados mostraron que no existían diferencias significativas en el puntaje posintervención de los grupos, las áreas en que se observaron mayores ganancias para el grupo experimental fueron: la categorización icónica, en la que se pide a los estudiantes categorizar objetos usando imágenes, y las analogías icónicas, en las que los alumnos también usaron imágenes para establecer relaciones entre objetos. En las ha- bilidades verbales orales (sin estímulo visual) las ganancias fueron inferiores, lo que confirma la importancia de usar material visual o concreto como un andamiaje efectivo para construir significado, en el caso de los niños más pequeños. Cabe recordar que todas estas actividades (categorizar, resolver adivinanzas, identificar conceptos absurdos o completar analogías) fueron ejercitadas durante las seis semanas de intervención, en forma tanto verbal oral como complementada con imágenes o material concreto. Ello favoreció la incorporación de las nuevas palabras de vocabulario al léxico de los alumnos.

Evidentemente, la efectividad de una estrategia de aprendizaje de vocabulario depende en gran medida de la duración de la intervención. Otro aspecto determinante es quién realiza la intervención. Tal como muestran Marulis y Neuman (2010), aspectos tales como la duración y la frecuencia de la intervención determinan el tamaño de efecto de la intervención. Uno de los factores más determinantes del éxito de la intervención, sostienen las autoras, es el perfil de la persona que capacita en el uso de la estrategia. En el caso del contexto preescolar, los mejores resultados se observan cuando quien capacita es un investigador, y los peores parecen darse cuando la intervención es transmitida por una educadora a sus pares. Estudios acerca del desarrollo profesional docente muestran que, en el caso de las maestras preescolares, es necesaria una mayor dosis de capacitación y apoyo para mejorar las habilidades de lenguaje oral, en comparación con otro tipo de habilidades, como, por ejemplo, el aprendizaje del alfabeto. Por otra parte, y pese a que sería esperable que intervenciones de mayor duración produjeran mejores resultados, la evidencia del metaanálisis realizado por Marulis y Neuman (2010) da cuenta de resultados mixtos, lo que se observó anteriormente en estudios realizados por Whitehurst et al. (1988). En el presente estudio, el hecho de que la capacitación y el seguimiento hayan sido realizados por el equipo investigador constituye un aspecto que podría asociarse a los buenos resultados, pero no es posible es- 
tablecer si la duración fue un factor importante. Se requiere de más estudios cuasiexperimentales que tomen en consideración estos factores propios de la intervención para establecer con mayor certeza si el impacto positivo del uso de la estrategia corresponde, en efecto, a su duración, o si el factor tiempo puede aislarse para determinar la efectividad de la lectura repetida interactiva en este contexto.

\section{Limitaciones y conclusiones}

El estudio que se presenta tiene dos limitaciones evidentes. Una de ellas es el tamaño de la muestra, que impide establecer mayores comparaciones y generalizar a partir de los resultados. Además de tratarse de solo dos cursos en dos jardines infantiles, estos tampoco son representativos de la población preescolar infantil chilena. Se trata de una muestra pequeña de un sector social altamente vulnerable, por lo que los resultados podrían variar si se incluyeran alumnos de otras zonas geográficas y de otros niveles socioeconómicos. En segundo lugar, la diferencia de dos meses de edad del grupo experimental respecto del grupo control puede ser una variable que afecte los resultados obtenidos, sobre todo considerando que se trata de una época del desarrollo infantil en la que los cambios en el desarrollo del lenguaje se observan de manera permanente. En tercer lugar, la duración de la intervención fue breve, lo que tampoco permite explorar con mayor profundidad el impacto de la misma en los estudiantes. Si bien se recabó información de tipo cualitativo a fin de evaluar la fidelización en la implementación de la lectura repetida interactiva, dichos datos no se incluyen en el presente análisis. No obstante, ellos revelan que, pese a que se les enseñó una estrategia de lectura novedosa y se les apoyó tanto en lo logístico (entrega de materiales, planificaciones, retroalimentación y actividades) como en lo pedagógico (mediante el modelamiento de las actividades que la lectura incluye), aún está muy inserto en el aula un patrón de diálogo menos sofisticado del lenguaje expresivo. Tampoco se utilizan preguntas de distinto nivel taxonómico (Tornero, Ramaciotti, Trufello y Valenzuela, 2015). El predominio de preguntas de tipo literal impide que los niños extiendan su conversación al comentar las lecturas o al usar las palabras nuevas aprendidas.

El énfasis de las educadoras en aspectos más vinculados a la gestión del aula (hacer callar a los niños, interrumpir para dar una instrucción no relacionada con la lectura del cuento, por ejemplo) impide en gran medida que la lectura repetida interactiva fomente un diálogo de mayor complejidad en el que los estudiantes tengan mayor autonomía para expresarse usando el lenguaje más libremente, que a su vez dé cuenta del propio pensamiento. Estos aspectos dan cuenta de que el hecho de capacitar y acompañar a las educadoras en la implementación de una estrategia novedosa para trabajar el lenguaje y la lectura no es siempre suficiente para producir un impacto en los alumnos. En cierta medida, la adquisición de la estrategia de lectura repetida interactiva se vería más beneficiada con un mayor conocimiento teórico y práctico de la importancia del tipo de diálogo que se produce en la etapa preescolar para el crecimiento del pensamiento y del lenguaje y cómo estos dos aspectos están tan íntimamente relacionados e inciden en el aprendizaje posterior de los alumnos.

Promover la lectura interactiva y dialogada en todos los niveles de la educación preescolar y escolar debería ser una prioridad de los sistemas escolares de países en los que, si bien se cuenta con bastante cobertura, aún se observa poco aprovechamiento del tiempo en el aula para actividades de alfabetización. Las ganancias que producen las instancias de lectura interactiva son especialmente importantes para aquellos niños que, como los participantes del presente estudio, carecen de experiencias lectoras en el hogar, que les impiden muchas veces incrementar su repertorio léxico, expresivo y lingüístico en general. 


\section{Anexo.}

\section{Ejemplo de Planificación para la Implementación de la Lectura Repetida Interactiva}

Sesión No. 2

Libro: Franklin es un mandón (Bourgeois, Clark y Salazar 2003)

Palabras a trabajar: terrible, actitud, proteger, justo, molesto.

Lunes: Conocimiento del cuento y su vocabulario.

\section{Parte I}

Cuente a los niños que ha llegado la "Hora del cuento", ese momento de la rutina tan especial y que han esperado por tanto rato. Recuerde que debe (Beuchat, 2006):

- Usar un tono de voz adecuado: natural, respetando los rasgos de la persona, sin exagerar. No se recomienda forzar tonos ni muy agudos ni muy graves.

- Contar el cuento con melodía y ritmo: es decir, debe mantener un ritmo y velocidad natural, ni muy rápido ni tan lento. Dependerá de aquello que esté contando: si es algo de suspenso, el ritmo descenderá; si es de alegría, se acelerará.

- Cuidar su respiración: es necesario aprender a usar la voz, de manera que no se canse; será un buen modelo de lectura. Debe utilizar las pausas conscientemente, de manera de separar, por ejemplo, lo que dicen los personajes que señala el narrador y mantener así el interés de quien lo escucha.

- Cuidar de mirar a todos los niños, cubriendo al grupo completo y a cada uno en particular: la mirada hacia quienes le escuchan juega un rol fundamental, pues debe captar la atención de los oyentes y a la vez transmitir parte de lo que se está leyendo. Idealmente debe captar la mirada de todos, produciendo un encuentro de miradas. Para ello, evite que el pelo caiga sobre los ojos, obstaculizando la mirada. Es una mirada de "barrido", que envuelve a los oyentes y los introduce en el mundo del relato, no se fija en un solo punto, sino que se desplaza lentamente de uno a otro; todos quienes escuchan deben sentirse incluidos. Si bien usted estará leyendo, por lo que el foco principal de su mirada debe estar en el texto leído, debe también incorporar el mirar a quienes lee. El haber leído en forma previa varias veces el texto favorece el despegarse del mismo para prestar atención a la audiencia. Esto al principio no es fácil, pero ¡ánimo!, el tener estas consideraciones presentes y practicarlas ayudan mucho a conseguir el objetivo.

Sugerimos que distribuya a los niños de la siguiente manera, de forma de contribuir a desarrollar una atmosfera especial.

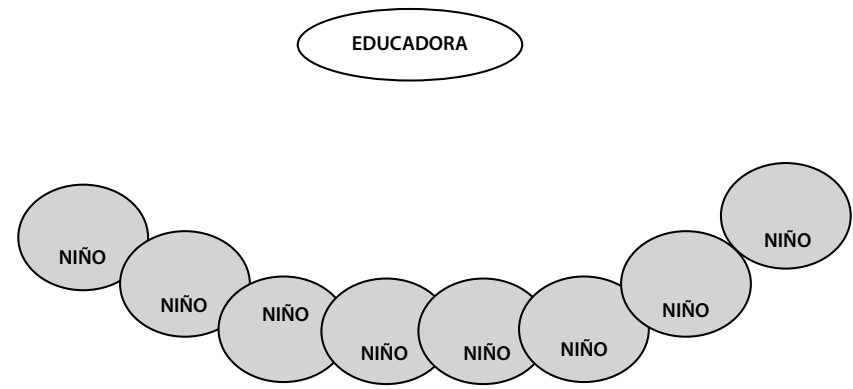


Tome el libro y muestre dónde está el título (sígalo con su dedo, indicando a los niños que ese es el título del cuento "Franklin es un mandón”). Luego indique que más abajo están los autores del cuento, que son quienes lo escribieron. En este caso, los autores son Paulette Bourgeois y Brenda Clark (muestre con su dedo en qué parte están ubicados los nombres de los autores). Indique que esa es la tapa o portada del cuento, y que el resto son las páginas que lo componen.

Vuelva a leer el título y pregunte a los niños ¿de qué creen ustedes que se tratará? Muestre a los niños la portada, para que contesten: ¿qué está haciendo Franklin? ¿Saben qué significa mandón? ¿Alguna vez les han dicho que son mandones?, buscando que los niños relacionen el título del cuento con sus experiencias previas.

\section{Parte II}

Luego invite a los niños a escuchar el cuento, señalando que hoy día aprenderán palabras nuevas:
a. terrible
b. actitud
c. proteger
d. justo
e. molesto

Para cada una de ellas, ofrecemos una "definición amigable", término propuesto por Beck, McKeown y Kucan (2002), para acercar al niño a la definición de la palabra.

a. Terrible: Algo que da mucho susto o produce sentimientos que no son agradables.

b. Actitud: Manera de comportarse con otras personas. Puede ser positiva o negativa.

c. Proteger: Cuidar un objeto o una persona para que no le pase nada malo.

d. Justo: Actuar pensando en el bien de todos.

e. Molesto: Estar muy enojado con otra persona.

Luego comience a contar el cuento y siga las consideraciones anteriormente entregadas.

\section{Parte III}

Al terminar de contar el cuento, formule a los niños las siguientes preguntas. Recuerde que es importante que dé tiempo para que piensen y luego contesten, y que debe escuchar las respuestas dadas, antes de formular la pregunta siguiente. Si requiere reformular la pregunta sugerida, para favorecer la comprensión del niño, según la respuesta dada, jhágalo! No olvide que el objetivo está en ayudar a comprender mejor el cuento. En un principio, los niños no saben cómo contestar, por lo que muestre usted cómo hacerlo. Es decir, formule la pregunta y luego conteste. 
- $\quad$ LLes ha pasado algo terrible?

- ¿Qué cosas les molestan?

- ¿Han protegido alguna vez a alguien?

- ¿Cómo lo protegieron?

Actividades de extensión:

Repase con los niños las palabras aprendidas y ayúdelos a recordar en qué contextos ellas aparecieron. Recuerde el trabajo que se realizó la semana anterior al jugar con las adivinanzas e invítelos a descubrir las siguientes adivinanzas. Recuerde leerlas con entonación y suspenso... jasí los niños harán su mayor esfuerzo en participar! Si los niños no descubren la respuesta, podrá guiar de manera que lleguen a ella.

a. Es que el pobre ve tan poco

que tampoco mira ya,

topa que topa que topa,

con la topa lo hallarás. (El topo)

b. Bolitas pequeñas,

de metal o de cristal,

mételas en el hoyo

y nunca perderás. (Las canicas)

c. Con ellos juego,

con ellos canto,

con ellos aprendo

en el jardín y el barrio. (Los amigos)

Miércoles: Compresión a través de preguntas.

Distribuya a los niños de manera de crear una atmósfera placentera para escuchar la lectura repetitiva interactiva

\section{Parte I}

Pregunte a los niños: ¿recuerdan cómo se llamaba este cuento que leímos el lunes?, ¿quién me puede contar de qué se trataba?, ¿con quién estaba Franklin?, ¿qué hacía que Franklin estuviera molesto?, buscando ayudar al niño a recordar la historia. 


\section{Parte II}

Cuente el cuento a los niños, tomando en consideración las indicaciones que ya se dieron para ello. Antes de iniciarlo, pregunte a los niños si recuerdan las palabras del cuento aprendidas el lunes (palabras de vocabulario enseñadas durante la primera parte de la intervención), pídales que las digan. Si no las recuerdan, ayúdelos dando una pequeña definición...

\section{Parte III}

Luego de leído el cuento, pregunte a los niños:

- ¿ ¿Les ha pasado que quieren que sus amigos hagan algo y ellos no quieren hacerlo?, ¿cuándo?

- ¿Cómo se han sentido?

- ¿Cómo creen ustedes que se sienten sus amigos si los obligan a hacer algo?

- ¿Se han disculpado con algún amigo cuando se han enojado?

- ¿Qué pasaría si siempre obligáramos a los amigos a hacer lo que nosotros queremos?

Actividades de extensión:

Invite a los niños a categorizar. Para ello, esta semana se trabajará con lápiz y papel. Utilizando láminas se categorizará entre artículos deportivos y juguetes. Presente las láminas a los niños (7 de juguetes y 7 de artículos deportivos). Luego de conversar sobre las láminas pegue todas las láminas desordenadas en el pizarrón de manera que todos los niños/as puedan ver (si prefiere, puede organizarlos en dos o tres grupos, y trabajar con los distintos elementos en grupo chico). Pregunte a los niños, ¿podemos agrupar estos objetos de alguna manera? Permita que algún niño lo haga, verbalicen en conjunto que los dos grupos organizados corresponden a dos agrupaciones distintas: juguetes y artículos deportivos. Las láminas se pueden pegar separadas por grupo de manera que los niños visualicen cada uno de los grupos.

Recuerde que es muy importante que verbalicen el criterio que se utilizó para armar las categorías, pues solo de esa manera estaremos ayudando al niño a utilizar el lenguaje para trabajar su pensamiento.

Láminas de juguetes: [imágenes]

Láminas de artículos deportivos: [imágenes]

Viernes: Reconstrucción de la historia guiada.

\section{Parte I}

Distribuya a los niños de manera de crear una atmósfera placentera para escuchar la Lectura Repetida Interactiva.

Diga a los niños: "Apuesto que se acuerdan del cuento que escuchamos el miércoles... ¿Cómo se llamaba? (recuerden juntos el título). Pregunte: ¿cuál era el problema que tenía Franklin? (quería jugar con sus propias 
reglas y mandaba a sus amigos, por lo que todos estaban enojados con él), y ¿cómo lo solucionó? (pidió disculpas a su amigo Oso y entendió que no debía forzar a sus amigos a jugar el juego que él quería).

\section{Parte II}

Invite a los niños a recontar la historia en conjunto. Para ello utilice las láminas especialmente preparadas para ello. Parta usted relatando el cuento, pero luego pida a los niños: ¿y qué pasó aquí?..., ¿y por qué?, ¿qué sucedió después? ... Utilice las palabras de vocabulario para contar el cuento, de manera de ayudar a los niños a que también lo usen.... Hagan inferencias sobre qué hubiese ocurrido si, por ejemplo, Franklin no hubiese sido mandón, o si no hubiese hablado con Oso para pedir disculpas, o si Oso no hubiese perdonado a Franklin...

De este modo, a través de estas tres presentaciones, está claro que los niños están construyendo una comprensión más rica, más detallada de la historia. Con la tercera lectura, los niños están utilizando claramente el diálogo analítico para ir más allá de lo que realmente sucedió en la historia.

Actividad de extensión:

Ya que el diálogo tomará gran parte de la sesión, no se proponen actividades de extensión para este día. La idea es que promueva el pensamiento divergente en los niños, con preguntas abiertas, que los inviten a pensar.

\section{Referencias}

Allor, J. H. y McCathren, R. B. (2003). Developing emergent literacy skills through storybook reading. Intervention in School and Clinic, 39(2), 72-79. DOI: 10.1177/10534512030390020201

Anderson, A., Anderson, J., Lynch,J., Shapiro, J.y Eun Kim,J. (2012). Extra-textual talk in shared book reading: A focus on questioning. Early Child Development and Care, 182(9), 1139-1154. DOI: 10.1080/03004430.2011.602189

Baker, L., Scher, D. y Mackler, K. (1997) Home and family influences on motivations for reading. Educational Psychologists, 32(2), 69-82. DOI:10.1207/s15326985ep32O2_2

Barrentine, S. (1996). Engaging with reading through interactive read-alouds. The Reading Teacher, 50(1), 36-43. Recuperado de https://www.jstor.org/stable/20201705

Beck, l., McKeown, M. y Kucan, L. (2002). Bringing words to life. Robust vocabulary instruction. Nueva York: The Guilford Press.

Beck, I.y McKeown, M. (2007). Increasing young low-income children's oral vocabulary repertoires through rich and focused instruction. The Elementary School Journal, 107(3), 251-271. DOI:10.1086/511706

Bedregal, P. (2006). Eficacia y efectividad en la atención de niños entre o y 4 años. En Foco, 79, 1-30. Recuperado de http://www.expansiva.cl/media/en_foco/documentos/19062006104824.pdf 
Beuchat, C. (2006). Narración oral y niños: una alegría para siempre. Santiago de Chile: Universidad Católica de Chile.

Blewitt, P., Rump, K. M., Shealy, S. E. y Cook, S. A. (2002). Shared book reading: When and how questions affect young children's word learning. Journal of Educational Psychology, 101(2), 294-304. DOI: 10.1037/a0013844

Bortnem, G. M. (2008). Teacher use of interactive read-alouds using nonfiction in early childhood classrooms. Journal of College Teaching and Learning, 5(12), 29-43. DOI: 10.19030/tlc.v5i12.1213

Bourgeois, P., Clark, B.y Salazar, M. (2003). Franklin es un mandón. Bogotá: Norma.

Burns, M., Griffin, P. y Snow, C. (eds.) (1999). Starting out right: A guide to promoting children's reading success. Washington: National Academy Press.

Bus, A. G., Van Ijzendoorn, M. H.y Pelligrini, A. D. (1995). Joint book reading makes for success in learning to read: A meta-analysis on intergenerational transmission of literacy. Review of Educational Research, 65, 1-21. DOI: 10.3102/00346543065001001

Cazden, C. B. (1986). Classroom discourse. En M. Wittrock (ed.), Handbook of research on teaching (3 ed.). Nueva York: Macmillan.

Connor, C. y Craig, H. (2006). African American preschoolers' language, emergent literacy skills, and use of African American English: A complex relation. Journal of Speech, Language, and Hearing Research, 49, 771792. DOI: 10.1044/1092-4388(2006/055)

Copenhaver-Johnson, J., Bowman, J. y Rietschlin, A. J. (2009). Culturally responsive read-alouds in first grade: Drawing upon children's languages and cultures to facilitate literary and social understandings. En J. C. Scott, D. Y. Straker y L. Katz (eds.), Affirming students' rights to their own language: Bridging language policies and pedagogical practices. Nueva York: Routledge \& NCTE.

Craig, H. K. Connor, C. M. y Washington, J. A. (2003). Early positive predictors of later reading comprehension for African American students: A preliminary investigation. Language, Speech, and Hearing Services in Schools, 34,31-43. Recuperado de http://hdl.handle.net/2027.42/83322

Cunningham, A. E. y Stanovich, K. E. (1997). Early reading acquisition and its relation to reading experience and ability ten years later. Developmental Psychology, 33, 934-945. DOI: 10.1037/0012-1649.33.6.934

Dickinson, D. (2001). Book reading in preschool classrooms: Is recommended practice common? En D. K. Dickinson y P. O. Tabors (eds.), Building literacy with language: Young children learning at home and school (pp. 175-203). Baltimore: Brookes.

Dickinson, D.y Smith, M. (1994). Long-term effects of preschool teachers'book readings on low-income children's vocabulary and story understanding. Reading Research Quarterly, 29(2), 105-122. DOI: 10.2307/747807

Dickinson, D. y Porche, M. (2011). Relation between language experiences in preschool classrooms and children's kindergarten and fourth-grade language and reading abilities. Child Development, 82(3), 870-886. DOI: 10.1111/j.1467-8624.2011.01576.x 
ISSN 0123-1294 | e-ISSN 2027-5358 | Educ.Educ. Vol. 21. No. 3 | Septiembre-diciembre de 2018 | pp. 409-432.

Universidad de La Sabana | Facultad de Educación

Dickinson, D., Golinkoff, R. y Hirsh-Pasek, K. (2010). Speaking out for language: Why language is central to reading development. Educational Researcher, 39(4), 305-310. Recuperado de https://www.jstor.org/ stable/27764601

Duke, N. K. y Kays, J. (1998). “Can I say 'Once upon a time'?” Kindergarten children developing knowledge of information book language. Early Childhood Research Quarterly, 13(2), 295-318. DOI: 10.1016/So8852006(99)80041-6

Echeverría, M., Herrera, M. O. y Segure, J. T. (1992). TEVI-R. Test de Vocabulario en Imágenes. Chile: Universidad de Concepción.

Eyzaguirre, B. y Fointaine, L. (2008). Las escuelas que tenemos. Santiago: Centro de Estudios Públicos.

Feitelson, D., Kita, B. y Goldstein, Z. (1986). Effects of listening to series stories on first graders' comprehension and use of language. Research in the Teaching of English, 20, 339-356. Recuperado de https://www.jstor. org/stable/40171092

Fitzgerald, J., Spiegel, D. L. y Cunningam, J. W. (1991). The relationship between parental literacy level and perceptions of emergent literacy.Journal of Reading Behavior, 23,191-212. DOI:10.1080/10862969109547736

Hargraves, A. C. y Sénéchal, M. (2000). A book reading intervention with preschool children who have limited vocabularies: The benefits of regular reading and dialogical reading. Early Childhood Research Quarterly, 15, 75-90. 10.1016/S0885-2006(99)00038-1

Hart, B. y Risley, T. R. (1995). Meaningful differences in the everyday experience of young American Children. Baltimore: Brookes.

Hoffman, J. L. (2011). Co-constructing meaning: Interactive literary discussions in kindergarten read-alouds. The Reading Teacher 65(3), 183-94. DOI:10.1002/TRTR.01025

Justice, L. M., Mashburn, A., Hambre, B. y Pianta, R. (2008). Quality of language and literacy instruction in preschool classrooms serving at-risk pupils. Early Childhood Research Quarterly, 23(1), 421-429. DOI: 10.1016/j.ecresq.2007.09.004

Justice, L. M. y Kaderavek, J. N. (2004). Embedded-explicit emergent literacy intervention I: background and description of approach. Language, Speech \& Hearing Services in Schools, 35, 201-221. DOI: 10.1044/01611461(2004/020)

Justice, L. M., Kaderavek, J. N., Fan, X., Sofka, A. y Hunt, A. (2009). Accelerating preschoolers' early literacy development through classroom-based teacher-child storybook reading and explicit print referencing. Language, Speech and Hearing Services in Schools, 4O(1), 67-85. DOI: 10.1044/0161-1461(2008/07-0098)

Kindle, K. J. (2009). Vocabulary development during read-alouds: Primary practices. The Reading Teacher, 63(3), 202-211. DOI: 10.1598/RT.63.3.3 
Kindle, K. (2011). Same book, different experience: a comparison of shared reading in preschool classrooms. Journal of Language and Literacy Education, 7(1), 13-34. Recuperado de https://files.eric.ed.gov/fulltext/ EJ1097102.pdf

Kucan, L. (2012). What is most important to know about vocabulary? The Reading Teacher, 65(6), 360-366. DOI: 10.1002/TRTR.01054

Larraín, A., Strasser, K. y Lissi, M. R (2012). Lectura compartida de cuentos y aprendizaje de vocabulario en edad preescolar: un estudio de eficacia. Estudios de Psicología, 33(3), 379-383. DOI: 10.1174/021093912803758165

Lawrence, J. y Snow, C. (2011). Oral discourse and reading. En M. L. Kamil, P. D. Pearson, E. Moje y P. Afflerbach (eds.), Handbook of Reading Research (vol. IV, pp. 320-337). Nueva York: Routledge.

Lennox, S. (2013). Interactive read-alouds. An avenue for enhancing children's language for thinking and understanding: A review of recent research. Early Childhood Education Journal, 41, 381-389. DOI: 10.1007/ s10643-013-0578-5

Leung, C. B. (2008). Preschoolers' acquisition of scientific vocabulary through repeated read-aloud events, retellings,andhands-onscienceactivities. ReadingPsychology,29, 165-193. DOI:10.1080/02702710801964090

Marulis, L. M. y Neuman, S. B. (2010). The effects of vocabulary intervention on young children's word learning: A meta-analysis. Review of Educational Research, 80(3), 300-335. DOI: 10.3102/0034654310377087

McGee, L.y Schickedanz, J. (2007). Repetead interactive read-alouds in preschool and kindergarten. The Reading Teacher, 6o(8), 742-751. DOI: 10.1598/RT.60.8.4

Mineduc (1998). Evaluación de programas de educación parvularia en Chile: resultados y desafios. Santiago: Ministerio de Educación.

Morrow, L., Tracey, D. y Renner Del Nero, J. (2011). Best practices in early literacy: Preschool, kindergarten and first grade. En L. Morrow, L. Gambrell y N. Duke (eds.), Best practices in literacy instruction (4 ed., pp. 67-95). Nueva York: Guilford Press.

Morrow, L. M. y Smith, J. K. (1990). The effects of group size on interactive storybook reading. Reading Research Quarterly, 25(3), 213-231. DOI: 10.2307/748003

Neslihan, D.y Simsek, O. (2014). Storybook reading of preschool teachers in the USA and Turkey. International Journal of Education and Research, 2(7), 41-54. Recuperado de http://www.ijern.com/journal/July-2014/o4.pdf

Neuman, S. B.y Celano, D. (2001). Access to print in low-income and middle-income communities: An ecological study of four neighborhoods. Reading Research Quarterly, 36, 8-26. DOI: 10.1598/RRQ.36.1.1

Neuman, S. B. (2003). From Rhetoric to Reality: The case for high quality prekindergarten. Phi Delta Kappan, 85(4), 286-291. DOI: 10.1177/003172170308500408

Noboa-Hidalgo, G.y Urzua, S. (2012). The effects of participation in public child care centers: Evidence from Chile. Journal of Human Capital, 6(1), 1-34. DOI: 10.1086/664790 
OECD - Organization for Economic Co-operation and Development (2013). Quality matters in early childhood education and care: Sweden 2013. M. Taguma, I. Litjens y K. Macowicki (comps.). París: OECD.

Orellana, P., Melo, C. y Fitzgerald, J. (2014). La relación entre la comprensión lectora silenciosa y sus subprocesos en niños con dificultades lectoras tempranas. Conferencia Internacional IRECE, Universidad Diego Portales, enero 8-10.

Orellana, P.y Melo, C. (2014). Ambiente letrado y estrategias didácticas en la educación preescolar chilena. Magis. Revista Internacional de Investigación en Educación, 6(13), 113-128. DOI: 10.11144/Javeriana.M6-13.ALED

Otto, B. (2008). Literacy development in early childhood. EE.UU.: Pearson.

Paris, A. H.y Paris, S. G. (2003). Assessing narrative comprehension in young children. Reading Research Quarterly, 38, 36-76. DOI: 10.1598/RRO..38.1.3

Paratore, J., Cassano, C.y Schickedanz, J. (2011). Support early (an later) literacy development at home and school: The long view. En M. Kamil, P. Pearson, E. Moje y P. Afflerbach (eds.), Handbook of Reading Research (4 ed., pp 107-135). Nueva York: Routledge.

Peralta, M. V. (2011). Early childhood education and public care policies in Chile. International Journal of Child Care and Education Policy, 5, 17-27. DOI: 10.1007/2288-6729-5-1-17

Pearson, P. D., Hiebert, E. H. y Kamil, M. L. (2007). Vocabulary assessment: what we know and what we need to learn. Reading Research Quarterly, 42(2), 282-295. DOI: 10.1598/RRO..42.2.4

Pianta, R. C. (2012). Handbook of Early Childhood Education. Nueva York: Guilford Press.

Renz, M., Valenzuela, M. F. y Yakuba, P. (2014). PHMS. Prueba para evaluar habilidades metaligüísticas de tipo semántico. Santiago: Universidad de Chile.

Reutzel, D. R. (2001). New thinking on read-aloud: New research helps you reap the biggest benefits from readaloud. Instructor (mayo-junio), 23-24.

Reznitskaya, A. y Anderson, R. (2002). The argument schema and learning to reason. En C. C. Block y M. Pressley (eds.), Comprehension instruction (pp. 319-334). Nueva York: Guilford.

Robbins, C. y Ehri, L. (1994). Reading storybooks to kindergartners helps them learn new vocabulary words. Journal of Educational Psychology, 86(1), 54-64. DOI: 10.1037/0022-0663.86.1.54

Rolla San Francisco, A., Arias, M. y Villers, R. (2005). Quality early childhood education in Costa Rica? Policy, practice, outcomes and challenges. Early Years, 25, 113-127. DOI: 10.1080/09575140500127956

Santoro, L. E., Chard, D. J., Howard, L. y Baker, S. K. (2008). Making the "very" most of classroom read-alouds to promote comprehension and vocabulary. The Reading Teacher, 61(5), 396-408. DOI: 10.1598/RT.61.5.4

Shatz, E. K. y Baldwin, R. S. (1986). Context clues are unreliable predictors of word meanings. Reading Research Quarterly, 21, 439-453. DOI: 10.2307/747615 
Silverman, R.y Crandell, J. (2010). Vocabulary practices in prekindergarten and kindergarten classrooms. Reading Research Quarterly, 45(3), 318-340. Recuperado de https://www.jstor.org/stable/27822890

Sipe, L. R. (2008). Storytime: Young children's literary understanding in the classroom. Nueva York: Teachers College Press.

Smolkin, L. B. y Donovan, C. A. (2001). The contexts of comprehension: information book read-alouds and comprehension acquisition. Elementary School Journal, 102, 97-122. Recuperado de https://www.jstor.org/ stable/1002203

Strasser, K. y Lissi, M. R. (2009). Home and instruction effects on emergent literacy in a sample of Chilean kindergarten children. Scientific Studies of Reading, 13, 175-204. DOI: 10.1080/10888430902769525

Strasser, K., Lissi, M. R.y Silva, M. (2009). Gestión del tiempo en 12 salas chilenas de Kindergarten: Recreo, colación y algo de instrucción. Psykhé, 18(1), 85-96. DOI: 10.4067/S0718-222820090001000242

Sulzby, E. y Teale, W. (1991). Emergent literacy. En R. Barr, M. Kamil, P. Mosenthal y P. D. Pearson (eds.), Handbook of Reading Research (vol. II, pp. 727-757). Nueva York: Longman.

Tornero, B., Ramaciotti, A., Truffello, A. y Valenzuela, F. (2015). Nivel cognitivo de las preguntas que formulan las educadoras de párvulos de segundo nivel de transición durante la hora del cuento. Educación y Educadores, 18(2), 261-283. DOI: 10.5294/edu.2015.18.2.5

Treviño, E. (2013). Calidad de la educación parvularia: las prácticas de clase y el camino a la mejora. Pensamiento Educativo, 50(1), 4O-62. DOI: 10.7764/PEL.50.1.2013.4

Urzúa, S. y Veramendi, G. (2011). The impact of out-of- home childcare centers on early childhood development. IDB Working Paper Series (IDB-WP-240).

Valenzuela, M. F. (2005). Instrucción temprana y alfabetización inicial. Estudio en niños de una comuna de Santiago. Tesis de Magister en Psicología Educacional, Universidad Católica de Chile, Escuela de Psicología, Santiago de Chile.

Van den Broek, P. (2001). The role oftelevision viewing in the development of reading comprehension. Washington: US Department of Education.

Villalón, M., Suzuki, E., Herrera, M. O. y Mathiesen, M. E. (2002). Quality of Chilean early childhood education from an international perspective. International Journal of Early Years Education, 10(1), 49-59. DOI: $10.1080 / 09669760220114845$

Wasik, B.y Bond, M. A. (2001). Beyond the pages of a book: Interactive book reading and language development in preschool classrooms. Journal of Educational Psychology, 93, 243-250. DOI: 10.1037/0022-0663.93.2.243

Whitehurst, G. J., Falco, F. L., Lonigan, C., Fischel, J. E., Valdez-Menchaca, M. C., De Baryshe, B. D.y Caulfield, M. (1988). Accelerating language development through picture-book reading. Developmental Psychology, 24, 552558. DOI:10.1037/0012-1649.24.4.552 
ISSN 0123-1294 | e-ISSN 2027-5358 | Educ.Educ. Vol. 21. No. 3 | Septiembre-diciembre de 2018 | pp. 409-432.

Universidad de La Sabana | Facultad de Educación

Wiseman, A. (2011). Interactive reads alouds: Teachers and students constructing knowledge and literacy together. Early Childhood Education Journal, 38, 431-438. DOI:10.1007/s10643-010-0426-9

Yoshikawa, H., Leyva, D., Snow, C. E. Treviño, E., Barata, C., Weiland, C., ... Arbour, M. C. (2015). Experimental impacts of a teacher professional development program in Chile on preschool classroom quality and child outcomes. Developmental Psychology, 51(3), 309-322. DOI: 10.1037/a0038785 